\title{
Secure Data Collection in Wireless Sensor Networks Using Randomized Dispersive Routes
}

\author{
Tao Shu, Marwan Krunz, and Sisi Liu \\ Department of Electrical and Computer Engineering \\ University of Arizona
}

\begin{abstract}
Compromised-node and denial-of-service are two key attacks in wireless sensor networks (WSNs). In this paper, we study data delivery mechanisms that can with high probability circumvent black holes formed by these attacks. We argue that classic multipath routing approaches are vulnerable to such attacks, mainly due to their deterministic nature. So once the adversary acquires the routing algorithm, it can compute the same routes known to the source, hence making all information sent over these routes vulnerable to its attacks. In this paper, we develop mechanisms that generate randomized multi-path routes. Under our designs, the routes taken by the "shares" of different packets change over time. So even if the routing algorithm becomes known to the adversary, the adversary still cannot pinpoint the routes traversed by each packet. Besides randomness, the generated routes are also highly dispersive and energyefficient, making them quite capable of circumventing black holes. We analytically investigate the security and energy performance of the proposed schemes. We also formulate an optimization problem to minimize the end-to-end energy consumption under given security constraints. Extensive simulations are conducted to verify the validity of our mechanisms.
\end{abstract}

Index Terms-Randomized multi-path routing, wireless sensor network, secure data delivery.

\section{INTRODUCTION}

\subsection{Motivations}

Of the various possible security threats encountered in a wireless sensor network (WSN), in this paper we are specifically interested in combating two types of attacks: compromised-node $(\mathrm{CN})$ and denial-of-service (DOS) [22]. In the $\mathrm{CN}$ attack, an adversary physically compromises a subset of nodes to eavesdrop information, whereas in the DOS attack, the adversary interferes with the normal operation of the network by actively disrupting, changing, or even paralyzing the functionality of a subset of nodes. These two attacks are similar in the sense that they both generate black holes: areas within which the adversary can either passively intercept or actively block information delivery. Due to the unattended nature of WSNs, adversaries can easily produce such black holes [1]. Severe $\mathrm{CN}$ and DOS attacks can disrupt normal data delivery between sensor nodes and the sink, or even partition the topology. A conventional cryptography-based security method cannot alone provide satisfactory solutions to these problems. This is because, by definition, once a node is compromised, the adversary can always acquire the encryption/decryption keys of that node, and thus can intercept any information passed through it. Likewise, an adversary can always

A preliminary version of this paper was presented at the IEEE INFOCOM 2009 Mini-Conference. This research was supported in part by NSF (under grants CNS-0721935, CNS-0627118, CNS-0325979, and CNS-0313234), Raytheon, and Connection One (an I/UCRC NSF/industry/university consortium). Any opinions, findings, conclusions, or recommendations expressed in this paper are those of the author(s) and do not necessarily reflect the views of the National Science Foundation. perform DOS attacks (e.g., jamming) even if it does not have any knowledge of the underlying crypto-system.

One remedial solution to these attacks is to exploit the network's routing functionality. Specifically, if the locations of the black holes are known a priori, then data can be delivered over paths that circumvent (bypass) these holes, whenever possible. In practice, due to the difficulty of acquiring such location information, the above idea is implemented in a probabilistic manner, typically through a two-step process. First, the packet is broken into $M$ shares (i.e., components of a packet that carry partial information) using a $(T, M)$-threshold secret-sharing mechanism such as the Shamir's algorithm [20]. The original information can be recovered from a combination of at least $T$ shares, but no information can be guessed from less than $T$ shares. Second, multiple routes from the source to the destination are computed according to some multi-path routing algorithm (e.g., [5], [8], [13], [24]). These routes are node-disjoint or maximally node-disjoint subject to certain constraints (e.g., min-hop routes). The $M$ shares are then distributed over these routes and delivered to the destination. As long as at least $M-T+1$ (or $T$ ) shares bypass the compromised (or jammed) nodes, the adversary cannot acquire (or deny the delivery of) the original packet.

We argue that three security problems exist in the above counter-attack approach. First, this approach is no longer valid if the adversary can selectively compromise or jam nodes. This is because the route computation in the above multi-path routing algorithms is deterministic in the sense that for a given topology and given source and destination nodes, the same set of routes are always 
computed by the routing algorithm. As a result, once the routing algorithm becomes known to the adversary (this can be done, e.g., through memory interrogation of the compromised node), the adversary can compute the set of routes for any given source and destination. Then the adversary can pinpoint to one particular node in each route and compromise (or jam) these nodes. Such an attack can intercept all shares of the information, rendering the above counter-attack approaches ineffective. Second, as pointed out in [24], actually very few node-disjoint routes can be found when the node density is moderate and the source and destination nodes are several hops apart. For example, for a node degree of 8 , on average only two node-disjoint routes can be found between a source and a destination that are at least 7 hops apart. There is also 30\% probability that no nodedisjoint paths can be found between the source and the destination [24]. The lack of enough routes significantly undermines the security performance of this multi-path approach. Last, because the set of routes is computed under certain constraints, the routes may not be spatially dispersive enough to circumvent a moderate-size black hole.

In this paper, we propose a randomized multi-path routing algorithm that can overcome the above problems. In this algorithm, multiple paths are computed in a randomized way each time an information packet needs to be sent, such that the set of routes taken by various shares of different packets keep changing over time. As a result, a large number of routes can be potentially generated for each source and destination. To intercept different packets, the adversary has to compromise or jam all possible routes from the source to the destination, which is practically not possible.

Because routes are now randomly generated, they may no longer be node-disjoint. However, the algorithm ensures that the randomly generated routes are as dispersive as possible, i.e., the routes are geographically separated as far as possible such that they have high likelihood of not simultaneously passing through a black hole. Considering the stringent constraint on energy consumption in WSNs, the main challenge in our design is to generate highly dispersive random routes at low energy cost. As explained later, such a challenge is not trivial. A naive algorithm of generating random routes, such as Wanderer scheme [2] (a pure randomwalk algorithm), only leads to long paths (containing many hops, and therefore, consuming lots of energy) without achieving good dispersiveness. Due to security considerations, we also require that the route computation be implemented in a distributed way, such that the final route represents the aggregate decision of all the nodes participating in the route selection. As a result, a small number of colluding/compromised nodes cannot dominate the selection result. In addition, for efficiency purposes, we also require that the randomized route selection algorithm only incurs a small amount of communication overhead.

\subsection{Contributions and Organization}

The key contributions of this work are as follows: 1. We explore the potential of random dispersion for information delivery in WSNs. Depending on the type of information available to a sensor, we develop four distributed schemes for propagating information "shares": purely random propagation (PRP), directed random propagation (DRP), non-repetitive random propagation (NRRP), and multicast tree-assisted random propagation (MTRP). PRP utilizes only one-hop neighborhood information and provides baseline performance. DRP utilizes two-hop neighborhood information to improve the propagation efficiency, leading to a smaller packet interception probability. The NRRP scheme achieves a similar effect, but in a different way: it records all traversed nodes to avoid traversing them again in the future. MTRP tries to propagate shares in the direction of the sink, making the delivery process more energy efficient.

2. We theoretically evaluate the goodness of these dispersive routes in terms of avoiding black holes. We conduct asymptotic analysis (i.e., assuming an infinite number of nodes) for the worst-case packet interception probability and energy efficiency under the baseline PRP scheme. Our results can be interpreted as the performance limit of PRP, and a lower-bound on the performance of the more advanced DRP, NRRP, and MTRP schemes. Our analysis helps us better understand how security is achieved under dispersive routing. Based on this analysis, we investigate the tradeoff between the random propagation parameter and the secret sharing parameter. We further optimize these parameters to minimize the end-to-end energy consumption under a given security constraint.

3 . We conduct extensive simulations to study the performance of the proposed schemes under more realistic settings. Our simulation results are used to verify the effectiveness of our design. When the parameters are appropriately set, all four randomized schemes are shown to provide better security performance at a reasonable energy cost than their deterministic counterparts. At the same time, they do not suffer from the type of attacks faced by deterministic multi-path routing.

The remainder of this paper is organized as follows. In Section 2, we elaborate on the design of the randomized multi-path routing mechanism. In Section 3, we analyze the performance of the baseline PRP scheme. Section 4 evaluates the performance of all four schemes using simulations. We overview related work in Section 5 and conclude the paper in Section 6.

\section{Randomized Multi-path Delivery}

\subsection{Overview}

As illustrated in Figure 1, we consider a 3-phase approach for secure information delivery in a WSN: secret sharing of information, randomized propagation of each information share, and normal routing (e.g., minhop routing) toward the sink. More specifically, when 


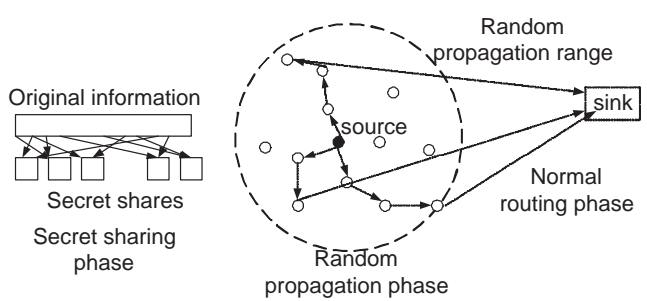

Fig. 1. Randomized dispersive routing in a WSN.

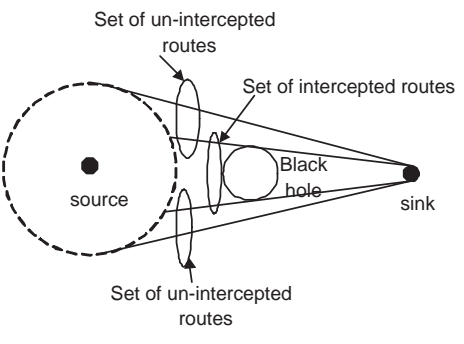

(a) Routes of higher dispersiveness

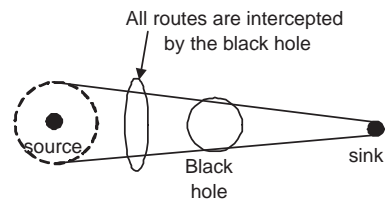

(b) Routes of lower dispersiveness
Fig. 2. Implication of route dispersiveness on bypassing the black hole.

a sensor node wants to send a packet to the sink, it first breaks the packet into $M$ shares according to a ( $T, M)$-threshold secret sharing algorithm, e.g., Shamir's algorithm [20]. Each share is then transmitted to some randomly selected neighbor. That neighbor will continue to relay the share it has received to other randomly selected neighbors, and so on. In each share, there is a TTL field, whose initial value is set by the source node to control the total number of random relays. After each relay, the TTL field is reduced by 1 . When the TTL value reaches 0 , the last node to receive this share begins to route it towards the sink using min-hop routing. Once the sink collects at least $T$ shares, it can reconstruct the original packet. No information can be recovered from less than $T$ shares.

The effect of route dispersiveness on bypassing black holes is illustrated in Figure 2, where the dotted circles represent the ranges the secret shares can be propagated to in the random propagation phase. A larger dotted circle implies that the resulting routes are geographically more dispersive. Comparing the two cases in Figure 2 , it is clear that the routes of higher dispersiveness are more capable of avoiding the black hole. Clearly, the random propagation phase is the key component that dictates the security and energy performance of the entire mechanism.

\subsection{Random propagation of Information Shares}

To diversify routes, an ideal random propagation algorithm would propagate shares as dispersively as possible. Typically, this means propagating the shares farther from their source. At the same time, it is highly desirable to have an energy-efficient propagation, which calls for limiting the number of randomly propagated hops. The challenge here lies in the random and distributed nature of the propagation: a share may be sent one-hop farther from its source in a given step, but may be sent back closer to the source in the next step, wasting both steps from a security standpoint. To tackle this issue, some control needs to be imposed on the random propagation process.

\subsubsection{Purely Random Propagation (Baseline Scheme)}

In PRP, shares are propagated based on one-hop neighborhood information. More specifically, a sensor node maintains a neighbor list, which contains the ids of all nodes within its transmission range. When a source node wants to send shares to the sink, it includes a TTL of initial value $N$ in each share. It then randomly selects a neighbor for each share, and unicasts the share to that neighbor. After receiving the share, the neighbor first decrements the TTL. If the new TTL is greater than 0 , the neighbor randomly picks a node from its neighbor list (this node cannot be the source node) and relays the share to it, and so on. When the TTL reaches 0 , the final node receiving this share stops the random propagation of this share, and starts routing it towards the sink using normal min-hop routing. The WANDERER scheme [2] is a special case of PRP with $N=\infty$.

The main drawback of PRP is that its propagation efficiency can be low, because a share may be propagated back and forth multiple times between neighboring hops. As shown in the analysis and simulations in subsequent sections, increasing the TTL value does not fully address this problem. This is because the random propagation process reaches steady state under a large TTL, and its distribution will no longer change even if the TTL becomes larger.

\subsubsection{Non-repetitive Random Propagation}

NRRP is based on PRP, but it improves the propagation efficiency by recording the nodes traversed so far. Specifically, NRRP adds a "node-in-route" (NIR) field to the header of each share. Initially, this field is empty. Starting from the source node, whenever a node propagates the share to the next hop, the id of the up-stream node is appended to the NIR field. Nodes included in NIR are excluded from the random pick at the next hop. This non-repetitive propagation guarantees that the share will be relayed to a different node in each step of random propagation, leading to better propagation efficiency.

\subsubsection{Directed Random Propagation}

DRP improves the propagation efficiency by using twohop neighborhood information. More specifically, DRP adds a "last-hop neighbor list" (LHNL) field to the header of each share. Before a share is propagated to the next node, the relaying node first updates the LHNL field with its neighbor list. When the next node receives the share, it compares the LHNL field against its own neighbor list, and randomly picks one node from its 
neighbors that are not in the LHNL. It then decrements the TTL value, updates the LHNL field, and relays the share to the next hop, and so on. Whenever the LHNL fully overlaps with or contains the relaying node's neighbor list, a random neighbor is selected, just as in the case of the PRP scheme. According to this propagation method, DRP reduces the chance of propagating a share back and forth by eliminating this type of propagation within any two consecutive steps. Compared with PRP, DRP attempts to push a share outward away from the source, and thus leads to better propagation efficiency for a given TTL value.

\subsubsection{Multicast Tree-assisted Random Propagation}

MTRP aims at actively improving the energy efficiency of random propagation while preserving the dispersiveness of DRP. The basic idea comes from the following observation of Figure 1: Among the 3 different routes taken by shares, the route on the bottom right is the most energy efficient because it is the shortest end-to-end path. So, in order to improve energy efficiency, shares should be best propagated in the direction of the sink. In other words, their propagation should be restricted to the right half of the circle in Figure 1.

Conventionally, directional routing requires location information of both the source and the destination nodes, and sometimes of intermediate nodes. Examples of location-based routing are the GPSR (Greedy Perimeter Stateless Routing) and LAR (Location-Aided Routing). Location information mainly relies on GPS in each node, or on some distributed localization algorithms. The high cost and the low accuracy of localization are the main drawbacks of these two methods, respectively.

MTRP involves directionality in its propagation process without needing location information. More specifically, it requires the sink to construct a multicast tree from itself to every node in the network. Such tree construction is not unusual in existing protocols, and is typically conducted by flooding a "hello" message from the sink to every node. Once the multicast tree is constructed, a node knows its distance (in hops) to the sink and the id of its parent node on the tree. We assume that each entry in the neighbor list maintained by a node has a field that records the number of hops to the sink from the corresponding neighbor. Under MTRP, the header of each share contains two additional fields: $\max _{h o p}$ and $\min _{\text {hop }}$. The values of these parameters are set by the source to $\max _{h o p}=n_{s}+\alpha_{1}$ and $\min _{h o p}=n_{s}-\alpha_{2}$, where $n_{s}$ is the hop count from the source to the sink, and $\alpha_{1}$ and $\alpha_{2}$ are non-negative integers with $\alpha_{1} \leq \alpha_{2}$. The parameter $\alpha_{1}$ controls the scope of propagation away from the sink, i.e., to the left half of the circle in Figure 1. The parameter $\alpha_{2}$ controls the propagation area toward the sink, i.e., the right half of the circle. A small $\alpha_{2}$ pushes the propagation of a share away from the center line connecting the source and the link and forces them to take the side path, leading to better dispersion.
Before a node begins to select the next relaying node, it first filters out neighbors that are in the LHNL, just as in DRP. Next, it filters out nodes whose hop count to the sink is greater than $\max _{\text {hop }}$ or smaller than $\min _{\text {hop }}$. The next relaying node will be randomly drawn from the remaining neighbors. In case the set of remaining nodes after the first step is empty, the second step will be directly applied to the entire set of neighbors.

\section{Asymptotic Analysis of the PRP SCHEME}

The random routes generated by the four algorithms in Section 2 are not necessarily node-disjoint. So a natural question is how good these routes are in avoiding black holes. We answer this question by conducting asymptotic analysis of the PRP scheme. Theoretically, such analysis can be interpreted as an approximation of the performance when the node density is sufficiently large. It also serves as a lower bound on the performance of the NRRP, DRP, and MTRP schemes. Note that the security analysis for the $\mathrm{CN}$ and DOS attacks are similar because both of them involve calculating the packet interception probability. For brevity, we only focus on the $\mathrm{CN}$ attack model. The same treatment can be applied to the DOS attack with a straightforward modification.

\subsection{Network and Attack Models}

We consider an area $S$ that is uniformly covered by sensors with density $\rho$. We assume a unit-disk model for the sensor communication, i.e., the transmitted signal from a sensor can be successfully received by any sensor that is at most $R_{h}$ meters away. Multi-hop relay is used if the intended destination is more than $R_{h}$ away from the source.

We assume that link-level security has been established through a conventional cryptography-based bootstrapping algorithm, i.e., consecutive links along an endto-end path are encrypted by symmetric link keys. So when a node $A$ wants to send a share to its neighbor $B$, it first encrypts the plaintext using link key $K_{A B}$ and then sends the ciphertext to $B$. When $B$ wants to forward the received share to its neighbor $C$, it decrypts the ciphertext using key $K_{A B}$, re-encrypts the plaintext using key $K_{B C}$, then sends it to $C$, and so on. In this way, the openness of the wireless media is eliminated: a node cannot decrypt a ciphertext overheard over the wireless channel if it is not the intended receiver. We also assume that a link key is safe unless the adversary physically compromises either side of the link.

The adversary has the ability to compromise multiple nodes. However, we assume that the adversary cannot compromise the sink and its immediate surrounding nodes. This assumption is reasonable because the sink's neighborhood is usually a small area, and can be easily physically secured by the network operator, 
e.g., by deploying guards or installing video surveillance/monitoring equipment. Such an assumption is also widely adopted in the literature, e.g., see [18], [23].

We assume that the black hole formed by the compromised nodes can be approximated by its circumcircle, i.e., the smallest circle that encompasses the shape of the black hole. Note that the schemes' operation does not depend on the shape of the black hole. The analysis of the security performance is conservative (i.e., the system is more secure than what it shows by analysis) under this assumption. We denote the circle, its center, and its radius by $E, e$, and $R_{e}$, respectively. During the WSN's operation, any end-to-end path that traverses through this circle is considered vulnerable to eavesdropping, i.e., information shares delivered over this path are all acquired by the adversary. In addition, we also assume that the area $S$ is sufficiently large such that the boundary effect of $S$ can be ignored in our analysis. We will consider the boundary effect in our simulations.

\subsection{Security Definition}

For a given source sensor node, the security provided by the protocol is defined as the worst-case (maximum) probability that for the $M$ shares of an information packet sent from the source, at least $T$ of them are intercepted by the black hole. Mathematically, this is defined as follows. Let the distance between the source $s$ and the sink $o$ be $d_{s}$. As shown in Figure 3, we define a series of $N+1$ circles co-centered at $s$. For the $i$ th circle, $1 \leq i \leq N$, the radius is $i R_{h}$. For circle 0 , its radius is 0 . These $N+1$ circles will be referred to as the N-hop neighborhood of $s$. More specifically, we say that a node is $i$ hops away from $s$ if it is located within the intersection between circles $i-1$ and $i$. We refer to this intersection as ring $i$. For an arbitrary share, after the random propagation phase, the id of the ring in which the last receiving node, say $w$, is located is a discrete random variable $\xi$ with state space $\{1, \ldots, N\}$. The actual path from $w$ to the sink is decided by the specific routing protocol employed by the network. Accordingly, different packet interception rates are obtained under different routing protocols. However, the route given by min-hop routing, which under high node density can be approximated by the line between $w$ and the sink, gives an upper bound on the packet interception rates under all other routing protocols. This can be justified by noting that min-hop routing tends not to distribute traffic over various intermediate nodes and only selects those nodes that are closest to the sink. As illustrated in Figure 3, this path-concentration effect makes min-hop routing have a smaller traversing area of the paths, and thus is more prone to packet interception, especially when compared to power-balancing routing protocols that build dispersive routes.

The worst-case scenario for packet interception happens when the points $s, e$, and $o$ in Figure 3 are collinear (the shaded region denotes the locations of $w$ for which the transmission from $w$ to $o$ using min-hop routing will be intercepted by $E$ ). Denote the distance between $e$ and $o$ by $d_{e}$. Given $d_{s}$ and $d_{e}$, when $s, e$, and $o$ are collinear, the shaded region attains its maximum area, and thus gives the maximum packet interception probability. For ring $i$, denote the area of its shaded portion by $S_{i}$. The interception probability for an arbitrary share of information is given by

$$
\begin{aligned}
P_{I} & =\sum_{i=1}^{N} \operatorname{Pr}\{\xi=i\} \frac{S_{i}}{\text { Area of ring } i} \\
& =\sum_{i=1}^{N} \operatorname{Pr}\{\xi=i\} \frac{S_{i}}{\pi i^{2} R_{h}^{2}-\pi(i-1)^{2} R_{h}^{2}} .
\end{aligned}
$$

Accordingly, the worst case probability that at least $T$ out of $M$ shares are intercepted by $E$ is given by

$$
P_{S}^{(\max )}=\sum_{k=T}^{M}\left(\begin{array}{c}
M \\
k
\end{array}\right) P_{I}^{k}\left(1-P_{I}\right)^{M-k}
$$

To proceed with the security analysis, we need to calculate the shaded area in each ring $S_{i}$ and the distribution of $\xi$.

\subsection{Derivation of the Packet Interception Area}

The derivation of $S_{i}$ falls into one of the following three cases:

Case 1: When $i R_{h} \leq \frac{R_{e} d_{s}}{d_{e}}$ (e.g., rings 1 to 3 in Figure $3)$, ring $i$ is completely covered by the shaded region. Therefore,

$$
S_{i}^{(\text {case 1) }}=\pi\left[i^{2}-(i-1)^{2}\right] R_{h}^{2}, 1 \leq i \leq\left\lfloor\frac{R_{e} d_{s}}{R_{h} d_{e}}\right\rfloor
$$

Case 2: When $(i-1) R_{h}<\frac{R_{e} d_{s}}{d_{e}}<i R_{h}$, as shown in Figure 4, ring $i$ is partially shaded. The shaded area of ring $i$ is the intersection of circle $i$ and the cone $C o D$ minus the area of circle $i-1$. The area of this intersection is composed of three components: the trapezoid $A_{1}$ $\left(B_{1} B_{2} B_{3} B_{4}\right)$, two circle segments $A_{2}$ (surrounded by arch $B_{1} B_{5} B_{2}$ and chord $B_{1} B_{2}$ ), and $A_{3}$ (surrounded by arch $B_{3} B_{6} B_{4}$ and chord $B_{3} B_{4}$ ). It can be shown that $A_{1}$ has a height $h_{A_{1}}=x_{1}-x_{2}$ where

$$
\begin{aligned}
& x_{1} \stackrel{\text { def }}{=} \frac{R_{e}^{2} d_{s}+\sqrt{R_{e}^{4} d_{s}^{2}-d_{e}^{2} R_{2}^{2} d_{s}^{2}+d_{e}^{4} i^{2} R_{h}^{2}-i^{2} d_{e}^{2} R_{h}^{2} R_{e}^{2}}}{d_{e}^{2}} \\
& x_{2} \stackrel{\text { def }}{=} \frac{R_{e}^{2} d_{s}-\sqrt{R_{e}^{4} d_{s}^{2}-d_{e}^{2} R_{2}^{2} d_{s}^{2}+d_{e}^{4} i^{2} R_{h}^{2}-i^{2} d_{e}^{2} R_{h}^{2} R_{e}^{2}}}{d_{e}^{2}} .
\end{aligned}
$$

The lengths of the two parallel edges of $A_{1}$ are given by

$$
\begin{aligned}
& l_{1}=2\left(-\frac{R_{e}}{\sqrt{d_{e}^{2}-R_{e}^{2}}} x_{1}+\frac{R_{e} d_{s}}{\sqrt{d_{e}^{2}-R_{e}^{2}}}\right) \\
& l_{2}=2\left(-\frac{R_{e}}{\sqrt{d_{e}^{2}-R_{e}^{2}}} x_{2}+\frac{R_{e} d_{s}}{\sqrt{d_{e}^{2}-R_{e}^{2}}}\right) .
\end{aligned}
$$




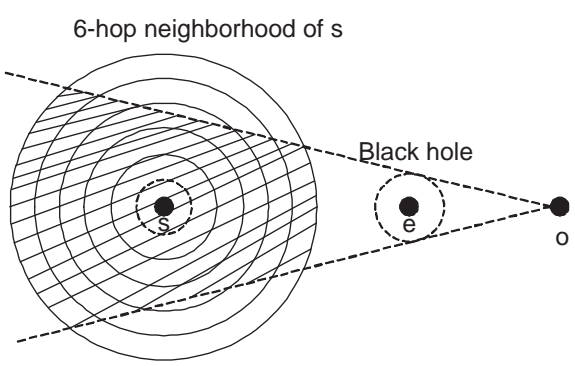

Fig. 3. Packet-interception area, a 6-hop random propagation example.

Therefore, the area of $A_{1}$ is given by

$$
S_{i}^{\left(A_{1}\right)}=\frac{\left(l_{1}+l_{2}\right) h_{A_{1}}}{2} .
$$

The area of $A_{2}$ and $A_{3}$ are given by

$$
\begin{array}{r}
S_{i}^{\left(A_{2}\right)}=\left(i R_{h}\right)^{2} \arctan \left(\frac{0.5 l_{1}}{x_{1}}\right)-0.5 x_{1} l_{1} \\
S_{i}^{\left(A_{3}\right)}=\left(i R_{h}\right)^{2} \arctan \left(-\frac{0.5 l_{2}}{x_{2}}\right)+0.5 x_{2} l_{2} .
\end{array}
$$

So the total shaded area in ring $i,\left\lceil\frac{R_{e} d_{s}}{R_{h} d_{e}}\right\rceil \leq i \leq\left\lfloor\frac{R_{e} d_{s}}{R_{h} d_{e}}+\right.$ $1\rfloor$, is given by

$$
S_{i}^{(\text {case 2) }}=S_{i}^{\left(A_{1}\right)}+S_{i}^{\left(A_{2}\right)}+S_{i}^{\left(A_{3}\right)}-\pi(i-1)^{2} R_{h}^{2},
$$

Case 3: When $(i-1) R_{h} \geq \frac{R_{e} d_{s}}{d_{e}}$, as shown in Figure 5, the shaded area in ring $i$ is the sum of the areas of two ring segments $B_{1}$ and $B_{2}$. Following a similar approach to case 2, the areas of $B_{1}$ and $B_{2}$ are approximated by

$$
\begin{gathered}
S_{i}^{\left(B_{1}\right)} \approx\left[i^{2}-(i-1)^{2}\right] R_{h}^{2} \arctan \left(\frac{0.5 l_{1}}{x_{1}}\right) \\
S_{i}^{\left(B_{2}\right)} \approx\left[i^{2}-(i-1)^{2}\right] R_{h}^{2} \arctan \left(-\frac{0.5 l_{2}}{x_{2}}\right)
\end{gathered}
$$

where $x_{1}, x_{2}, l_{1}$, and $l_{2}$ are given by (4) through (7), with $i$ referring to the ring being calculated. So the total shaded area in ring $i$ is

$$
S_{i}^{(\text {case } 3)}=S_{i}^{\left(B_{1}\right)}+S_{i}^{\left(B_{2}\right)}, \quad i \geq\left\lceil\frac{R_{e} d_{s}}{R_{h} d_{e}}+1\right\rceil
$$

\subsection{Derivation of Packet Interception Probability}

We derive the distribution of $\xi$ in this section. For a given share of information, its random propagation process can be modeled as a random walk, which is described by the $(N+1)$-state discrete-time Markov chain in Figure 6. A state in this Markov chain denotes the id of the ring that the share is at during the random propagation process. We first notice that the transition probability $P_{10}=0$. This is because in the random propagation process, the information share will never be relayed back to the source node. In addition, we note that the state $N$ is an absorbing state $\left(P_{N, N}=1\right.$ and $\left.P_{N, N-1}=0\right)$. This is because an information share takes totally $N$ hops in

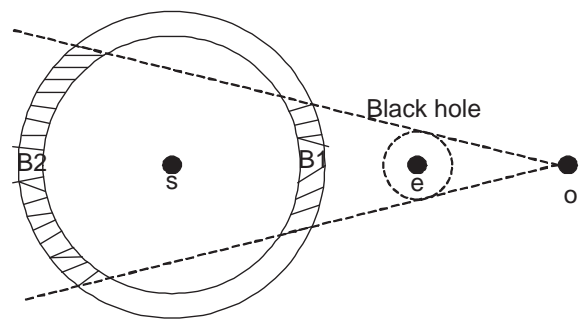

Fig. 5. Packet-interception area: case 3.

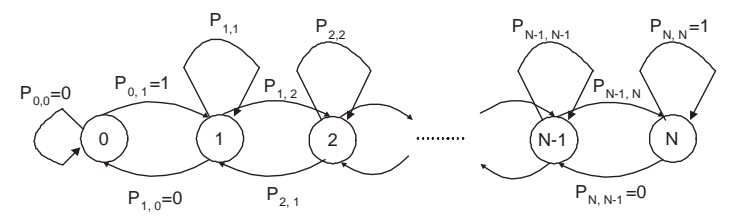

Fig. 6. $(N+1)$-state Markov chain for the random walk.

the random propagation phase, and thus it can reach as the farthest ring $N$. Furthermore, it is trivial to see that $P_{0,1}=1$ and $P_{0,0}=0$. The transition probability at other states can be calculated as follows.

Suppose that after the current hop, the share of information reaches at ring $i$, where $2 \leq i \leq N-1$. Let the location of the node that receives this share of information be $w$, and denote the one-hop neighborhood of $w$ as circle $O_{w}$ (This is the circle centered at $w$ and with a radius of $R_{h}$ ). As shown in Figure 7, the next hop from $w$ has three possibilities:

Case 1: Node $w$ picks a node in region $R_{1}$ as the next hop to relay the share. Region $R_{1}$ is defined as $R_{1}=$ $O_{w} \backslash$ Circle $i$, where the operation $A \backslash B$ denotes $A-A \cap B$. This case corresponds to the transition from state $i$ to $i+1$ in the random walk. We use Figure 8 to illustrate the calculation of the area of $R_{1}$. Given the distance from $w$ to $o$ be $d$, where $(i-1) R_{h}<d<i R_{h}$, the area of $R_{1}$ is the difference between the pies $G_{1}$ (the area surrounded by the arch $A B C$ and the edges $w A$ and $w C$ ) and $G_{2}$ (surrounded by arch $A D C$ and the edges $w A$ and $w C$ ). The area of $G_{1}$ is given by

$$
S_{G_{1}}=R_{h}^{2} \arcsin \left(\frac{\sqrt{i^{2} R_{h}^{2}-y^{2}}}{R_{h}}\right)
$$

where

$$
y=\frac{d^{2}+\left(i^{2}-1\right) R_{h}^{2}}{2 d}
$$

The area of $G_{2}$ is given by

$$
S_{G_{2}}=i^{2} R_{h}^{2} \arcsin \left(\frac{\sqrt{i^{2} R_{h}^{2}-y^{2}}}{i R_{h}}\right)-2 S_{\triangle A w s}
$$

where $S_{\triangle A w s}$ is the area of the triangle $A w s$ and can be calculated according to Heron's Formula:

$$
S_{\triangle A w s}=\sqrt{p\left(p-i R_{h}\right)(p-d)\left(p-R_{h}\right)}
$$




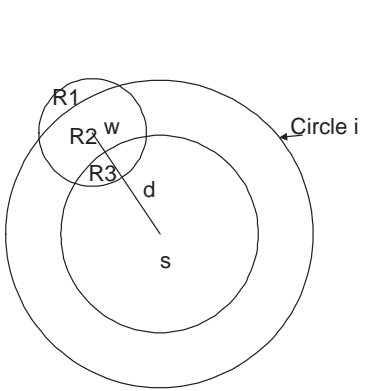

Fig. 7. Possibilities of the next hop.

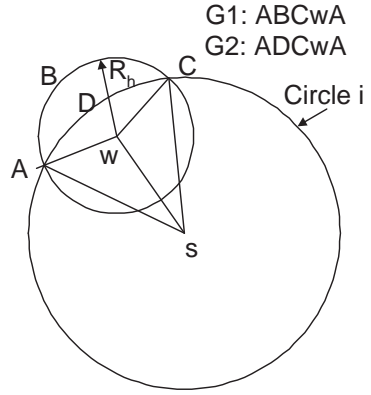

Fig. 8
$P_{i, i+1}$.

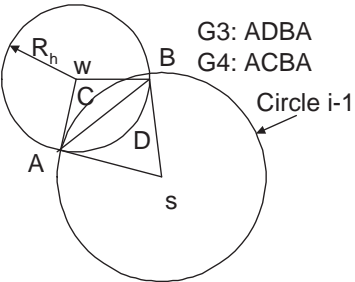

Fig. 9. Calculation of

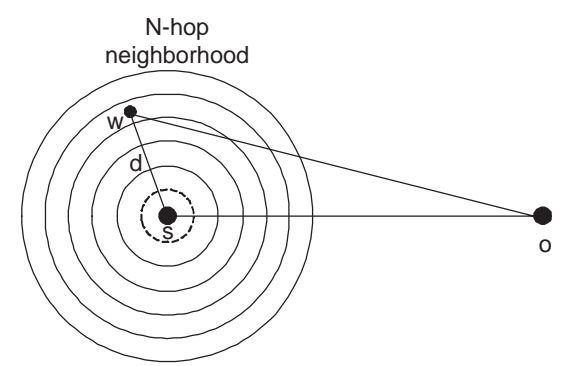

Fig. 10. The total transmission distance after random propagation. where $p=\frac{(i+1) R_{h}+d}{2}$ is half of the perimeter of the triangle.

Given that $(i-1) R_{h} \leq d \leq i R_{h}$, the conditional probability density function (pdf) of $d$ is given by

$f_{d}\left(d \mid(i-1) R_{h} \leq d \leq i R_{h}\right)= \begin{cases}\frac{2 d}{(2 i-1) R_{h}^{2}} & \text { for }(i-1) R_{h} \leq d \\ 0 & \text { otherwise }\end{cases}$

Therefore the transition probability $P_{i, i+1}$ can be calculated according to the probability theorem:

$$
P_{i, i+1}=\frac{1}{\pi R_{h}^{2}} \int_{(i-1) R_{h}}^{i R_{h}}\left(S_{G_{1}}(d)-S_{G_{2}}(d)\right) \frac{2 d}{(2 i-1) R_{h}^{2}} d d
$$

where $S_{G_{1}}$ and $S_{G_{2}}$ are written as functions of $d$.

Case 2: Node $w$ picks a node in region $R_{3}$ as the next hop to relay the share. The region $R_{3}$ is defined as $R_{3}=$ $O_{w} \cap$ Circle $i-1$. This case corresponds to the transition from state $i$ to $i-1$ in the random walk process. As shown in Figure 9, given the distance from $w$ to $o$ is $(i-1) R_{h}<d<i R_{h}$, the area of $R_{3}$ is the sum of the areas $G_{3}$ (surrounded by the arch $A D B$ and the chord $A B$ ) and $G_{4}$ (surrounded by the arch $A C B$ and the chord $A B)$.

The area of $G_{3}$ is given by

$$
\begin{array}{r}
S_{G_{3}}=R_{h}^{2} \arcsin \left(\frac{\sqrt{(i-1)^{2} R_{h}^{2}-y^{\prime 2}}}{R_{h}}\right) \\
-\left(d-y^{\prime}\right) \sqrt{(i-1)^{2} R_{h}^{2}-y^{\prime 2}}
\end{array}
$$

where $y^{\prime}=\frac{\left(i^{2}-2 i\right) R_{h}^{2}+d^{2}}{2 d}$. The area of $G_{4}$ is given by

$$
\begin{aligned}
S_{G_{4}}=(i-1)^{2} R_{h}^{2} \arcsin & \left(\frac{\sqrt{(i-1)^{2} R_{h}^{2}-y^{\prime 2}}}{(i-1) R_{h}}\right) \\
& -y^{\prime} \sqrt{(i-1)^{2} R_{h}^{2}-y^{\prime 2}}
\end{aligned}
$$

Following a similar argument in case 1, the transition probability $P_{i, i-1}$ is calculated as

$$
P_{i, i-1}=\frac{1}{\pi R_{h}^{2}} \int_{(i-1) R_{h}}^{i R_{h}}\left(S_{G_{3}}(d)+S_{G_{4}}(d)\right) \frac{2 d}{(2 i-1) R_{h}^{2}} d d
$$

Case 3: Node $w$ picks a node in region $R_{2}$ as the next hop to relay the share, where $R_{2}=O_{w} \backslash(R 1 \cup R 3)$. This corresponds to the situation that the information share will stay in ring $i$ after the next hop relay. Obviously the transition probability $P_{i, i}=1-P_{i, i+1}-P_{i, i-1}$.

$\leq i$ R When $i=1$, the calculation of $P_{1,2}$ follows exactly the same analysis as in Case 1, i.e., using (20). There will not be case 3 when $i=1\left(P_{1,0}=0\right)$. Therefore the transition probability $P_{1,1}=1-P_{1,2}$.

Denote the transition probability matrix of the Markov chain in Figure 6 by $\mathbf{P}$. The element of $\mathbf{P}$ can be numerically calculated according to above analysis. To calculate the distribution of $\xi$, we compute the N-step transition probability matrix by conducting the matrix power operation $\mathbf{P}^{N}$. The first row of the matrix $\mathbf{P}^{N}$ gives the probability mass vector of $\xi$. Substituting (3), (11), (14), and the distribution of $\xi$ into (1), the worst case packet interception probability is obtained.

\subsection{Energy Efficiency of the Random Propagation}

We assume that the energy consumption for delivering one bit over one hop is a constant $q$. Then the average energy consumption for delivering one packet from source $s$ to sink $o$ depends on the average length (in hops) of the route. Note that each random route consists of two components. The first is a fixed Nhop component attributed to the random propagation operation. The second component involves sending the share from the last random relay node, i.e., $w$, to the sink $o$ using a normal single path routing. Under the asymptotic assumption, when min-hop routing is used, the ratio between the number of hops from $w \rightarrow o$ and from $s \rightarrow o$ can be approximated by the ratio of the lengths of these two paths. This ratio can be calculated as follows.

Suppose $w$ is located in the $i$ th ring (see Figure 10). Let the distance between $w$ and $s$ be $(i-1) R_{h} \leq d \leq i R_{h}$. Given that the angle between $s w$ and $s o$ be $\theta$, the distance between $w$ and $o$ is given by

$$
d_{w o}^{(i)}(d, \theta)=\sqrt{d^{2}+d_{s}^{2}-2 d d_{s} \cos \theta}
$$

Due to the symmetry of the random propagation on all direction, $\theta$ uniformly distributed between 0 and $2 \pi$. 
Therefore the average distance while taking all directions into consideration is given by

$$
d_{w o}^{(i)}(d)=\int_{0}^{2 \pi} \frac{1}{2 \pi} \sqrt{d^{2}+d_{s}^{2}-2 d d_{s} \cos \theta} d \theta
$$

The average distance between $w$ and $o$ given that $(i-$ 1) $R_{h} \leq d \leq i R_{h}$ is given by

$d_{w o}^{(i)}=\int_{(i-1) R_{h}}^{i R_{h}} \int_{0}^{2 \pi} \frac{d}{(2 i-1) \pi R_{h}^{2}} \sqrt{d^{2}+d_{s}^{2}-2 d d_{s} \cos \theta} d \theta d d$

Therefore, the unconditionally average distance between $w$ and $o$ is given by the weighted sum of $d_{w o}^{(i)}$ 's with weights $\operatorname{Pr}\{\xi=i\}$, i.e.,

$$
\bar{d}_{w o}=\sum_{i=1}^{N} d_{w o}^{(i)} \operatorname{Pr}\{\xi=i\}
$$

where the distribution of $\xi$ has been obtained in Section 3.4. When min-hop routing is used in the third phase, the number of hops from $s$ to $o$ can be approximated by $d_{s} / R_{h}$. Let the lengths of an information packet and a share generated by the secret sharing algorithm be $L_{p}$ and $L_{s}$, respectively. Note that in general $L_{s} \geq \frac{L_{p}}{M}$, because a share contains a header and other redundant information of its original packet. To account for this segmentation overhead, let the extra bits of a share be a fraction, say $\alpha$, of the length of the original packet, i.e., $L_{s}=\frac{L_{p}}{M}+\alpha L_{p}$. Under this notation, the average energy consumptions for delivering one information packet using PRP can be calculated as follows:

$$
Q^{(\mathrm{PRP})}=M L_{s}\left(N+\frac{\bar{d}_{w o}}{R_{h}}\right) q=(1+M \alpha) L_{p}\left(N+\frac{\bar{d}_{w o}}{R_{h}}\right) q .
$$

\subsection{Energy-optimal Secret Sharing and Random Propagation}

In this section, we consider the problem of deciding the parameters for secret sharing $(M)$ and random propagation $(N)$ to achieve a desired security performance. To obtain the maximum protection of the information, the threshold parameter should be set as $T=M$. Then, increasing the number of propagation steps $(N)$ and increasing the number of shares a packet is broken into $(M)$ has a similar effect on reducing the message interception probability. Specifically, to achieve a given $P_{S}^{(\max )}$ for a packet, we could either break the packet into more shares but restrict the random propagation of these shares within a smaller range, or break the packet into fewer shares but randomly propagate these shares into a larger range. Therefore, when the security performance is concerned, a tradeoff relationship exists between the parameters $M$ and $N$. On the other hand, although different combinations of $M$ and $N$ may contribute to the same $P_{S}^{\max }$, their energy cost may be different, depending on the parameters $L_{s}, L_{p}$, and $q$. This motivates us to include their energy consumption into consideration when deciding the secret sharing and random propagation parameters: We can formulate an optimization problem to solve for the most energyefficient combination of $M$ and $N$ subject to a given security constraint. Formally, this is given as follows

$$
\begin{aligned}
\operatorname{minimize} & Q^{(\mathrm{PRP})}(M, N) \\
\text { s.t. } & P_{s}^{(\max )}(M, N) \leq P_{S}^{(r e q)} \\
& 1 \leq M \leq M_{\max } \\
& 1 \leq N \leq N_{\max }
\end{aligned}
$$

where $M$ and $N$ are variables and $P_{S}^{(r e q)}$ is the given security requirement. The upper bounds, $M_{\max }$ and $N_{\text {max }}$, are dictated by practical considerations such as the hardware or energy constraints. Because the range of $M$ and $N$ that are of practical interest is not large, e.g., at most few of tens, the space of feasible $(M, N)$ is moderate. Thus the optimal $\left(M^{o}, N^{o}\right)$ can be solved by the exhaustive search algorithm.

\subsection{Numerical Examples}

In this section, we use numerical examples to give a sense of the typical security performance of the PRP scheme. In all calculations, we assume that the secret sharing threshold $T=M$ and the hop range $R_{h}=10$ meters.

\subsubsection{Impact of Geometric Parameters}

Figure 11 plots the packet interception probability as a function of the black hole size under various combinations of $N$ and $M$. It is clear that the message is more likely to be intercepted when the black hole becomes larger, but increasing either $N$ or $M$ helps to reduce the interception rate. It is also noted from the crossing between curves that there is no absolute winner between increasing $N$ and increasing $M$ to reduce the interception probability. In the low-interception-probability regime, increasing $M$ gives better performance, while in the high-interception regime, increasing $N$ becomes better. This can be explained as follows: An increase in $N$ helps to propagate information shares more dispersively, thus reducing the interception probability of each share $\left(P_{I}\right)$. Increasing $M$ does not affect the interception probability of the share, but the black hole needs to collect more shares to recover a packet. From (2), it is clear the latter takes effect as the exponent while the former is on the base. When $P_{I} \ll 1$, a larger exponent provides faster decay of the probability than reducing the base, and vice versa.

We plot the packet interception probability as a function of the black hole location in Figures 12. It is clear that the closer the black hole to the sink, the larger the interception probability. This is in line with the manyto-one data collection paradigm in WSNs. For example, if the sink is compromised, then all packets will be intercepted by the adversary (no effective counter-attack measure exists in this case). 


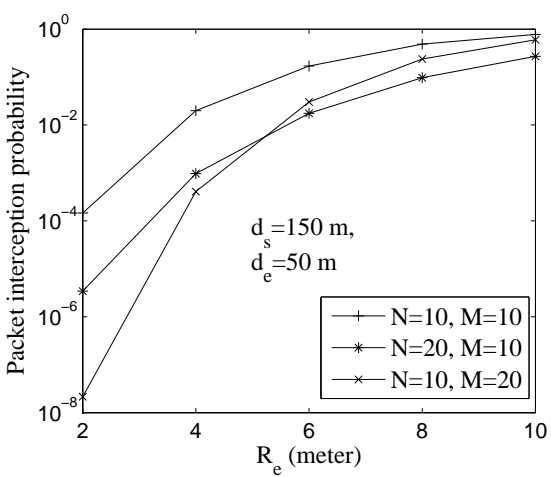

Fig. 11. Packet interception probability vs. black hole size.

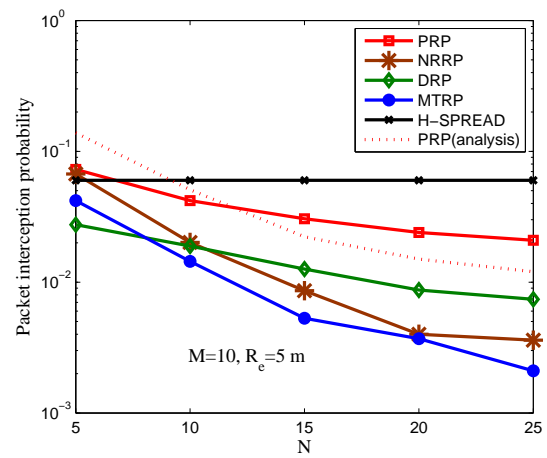

Fig. 14. Packet interception prob. vs. $N$.

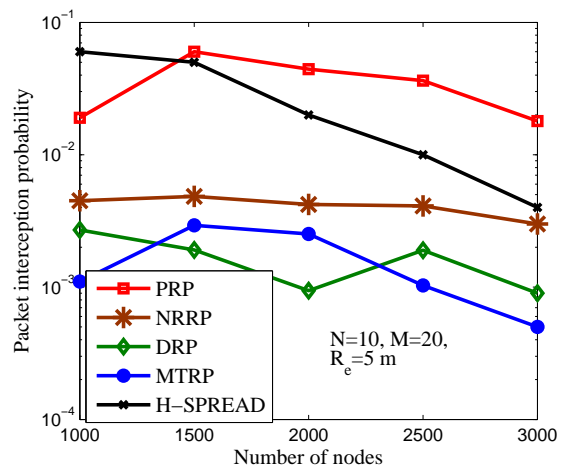

Fig. 17. Packet interception prob. vs. number of nodes.

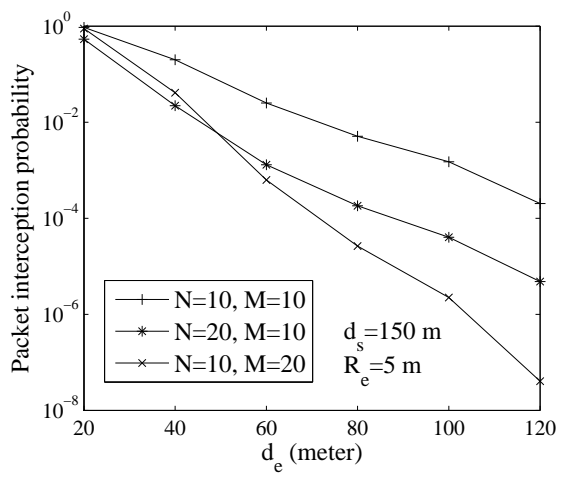

Fig. 12. Packet interception probability vs. black hole location.

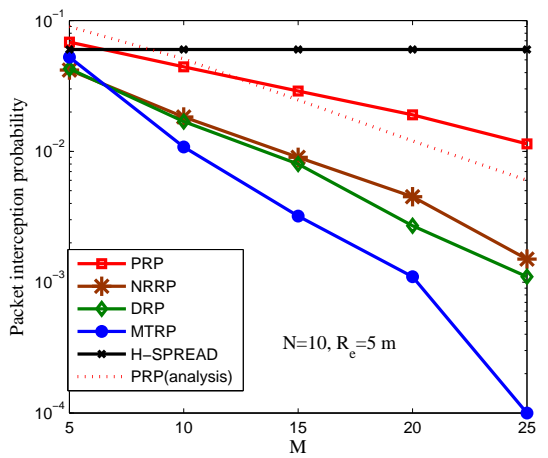

Fig. 15. Packet interception prob. vs. $M$.

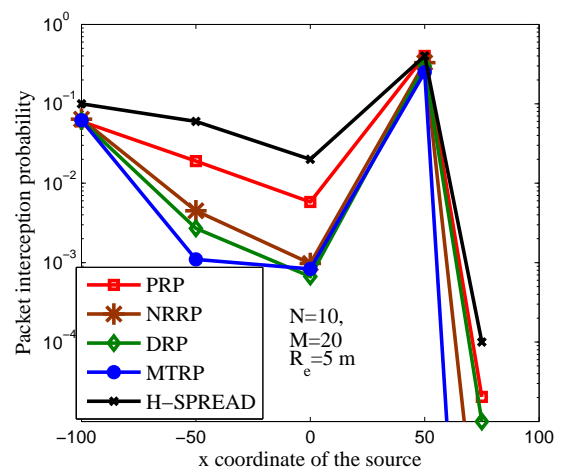

Fig. 18. Packet interception prob. at Fig. 19. Hop count of route vs. $N$. different source location. different $(N, M)$. $R_{e}$.

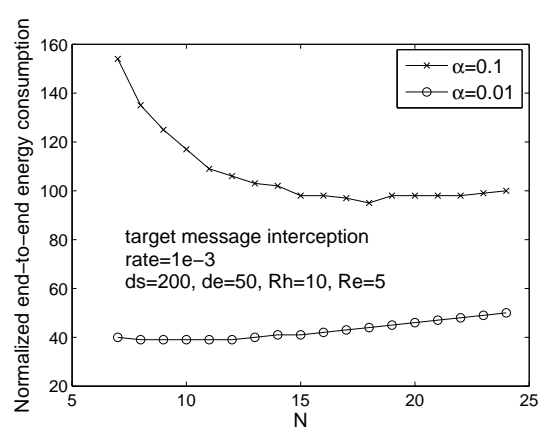

Fig. 13. Energy consumption under

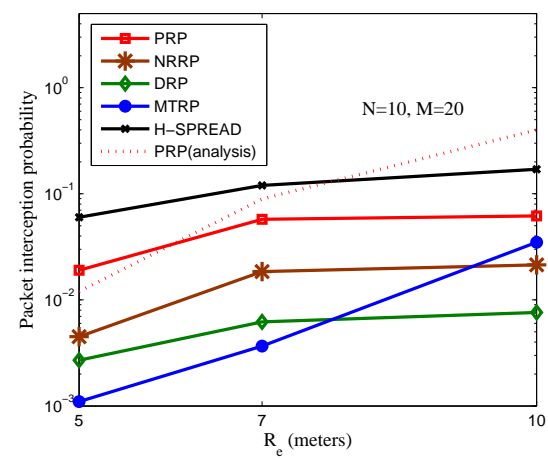

Fig. 16. Packet interception prob. vs.

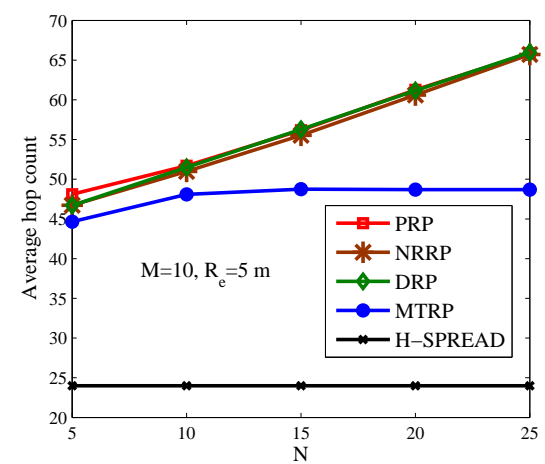

\subsubsection{Optimization of $N$ and $M$}

In Figure 13, for a target message interception probability of $10^{-3}$, we show the impact of the segmentation overhead $\alpha$ on the energy-optimal value of $(N, M)$. We fix the distance between the source node and the sink, but vary the segmentation overhead between two levels: high $(\alpha=10 \%)$ and low $(\alpha=1 \%)$. The energy consumption is normalized by $L_{p} q$, i.e., the amount of energy for transmitting the packet for one hop. Note that in this figure, only $N$ is shown and the corresponding $M$ satisfying the security requirement is omitted. Every point on both curves achieves the same target message interception probability. It shows that when the segmentation over- head becomes high, the optimization will favor a larger range of random propagation and a smaller breaking of the information $\left(\left(N^{o}=10, M^{o}=28\right)\right.$ when $\alpha=1 \%$, and $\left(N^{o}=17, M^{o}=16\right)$ when $\left.\alpha=10 \%\right)$. This can be explained by noting that under higher segmentation overhead, the delivery of each piece becomes more expensive in terms of energy consumption. Therefore, the best way is to partition the message into less number of piece, but propagate each piece into further distance. These results can serve as a guide in determining suitable $M$ and $N$ in the protocol's operation. We will evaluate the actual effect of this optimization using simulation in the next section. 


\section{Simulation Studies}

\subsection{Simulation Setup}

In this section, we use simulation to evaluate the performance of PRP, NRRP, DRP, and MTRP under more realistic settings. To better understand the capability of these randomized multi-path routing algorithms in bypassing black holes, we also compare their performance against a deterministic counterpart, H-SPREAD [10], which generates node-disjoint multi-path routes to combat $\mathrm{CN}$ attack in WSNs.

We consider a a $200 \mathrm{~m} \times 200 \mathrm{~m}$ field that is uniformly covered by sensors. The center of this square is the origin point. All coordinates are in the unit of meters. The sink and the center of the black hole are placed at $(100,0)$ and $(50,0)$, respectively. The transmission range of each sensor is $R_{h}=10 \mathrm{~m}$. For MTRP, we set the parameters $\alpha_{1}=0$ and $\alpha_{2}=5$. In all simulations, after the random propagation phase, each secret share is delivered to the sink using min-hop routing. Each simulation result is averaged over 50 randomly generated topologies. For each topology, 1000 information packets are sent from the source node to the sink. Our simulation results indicate that the nodes' locations have a significant impact on the absolute value of the packet interception probability of a given scheme. As a result, we emphasize that when reading the simulation results presented below, the absolute value of the mean performance is not as useful as the relative performance ranking between various schemes, and also not as useful as the general trend in performance. Because all comparisons made in our simulations are based on 50 common topologies, this common ground for comparison ensures that our results preserve the actual relative performance between various schemes.

\subsection{Simulation Results}

\subsubsection{Single-source Case}

We first fix the location of the source node at $(-50,0)$. In Figures 14 and 15, we plot the packet interception probability as a function of the TTL value $(N)$ and the number of shares $(M)$ that each packet is broken into, respectively. The packet interception probability calculated according to our asymptotic analytical model for PRP is also plotted in the same figure for comparison. These figures show that increasing $N$ and $M$ helps reduce the packet interception probability for all proposed schemes. However, for a sufficiently large $N$, e.g., $N=20$ in Figure 14, the interception probability will not change much with a further increase in $N$. This is because the random propagation process has reached steady state. It can also be observed that, in all cases, the packet interception probabilities under the DRP, NRRP, and MTRP schemes are much smaller than that of the baseline PRP scheme, because their random propagations are more efficient. In addition, when $N$ and $M$ are large, all four randomized algorithms achieve smaller packet interception probabilities than the deterministic H-SPREAD scheme. In many cases, the gap is more than one order of magnitude. The poor performance of H-SPREAD is due to the small number of node-disjoint routes that can be found by the algorithm when the source is far away from the sink (15 hops apart in our simulation), and the fact that these routes may not be dispersive enough. Increasing $M$ does not change the number of routes the algorithm can find, so it does not help in reducing the interception probability for H-SPREAD. Furthermore, it can be observed that the simulated performance for PRP is reasonably close to its theoretical performance, especially in the medium packet-interception-probability regime (i.e., $0.01 \leq P_{S} \leq 0.1$ ). This clearly demonstrates that the sample topologies used in our simulations are representative and sufficient, and the simulation results do represent the general performance trend. When the packet interception probability is high $\left(P_{S}>0.1\right)$ or low $\left(P_{S}<0.01\right)$, the gap between the theoretical and simulated results becomes more significant. The overlyoptimistic behavior of the analytical model in the low $P_{S}$ regime is due to ignoring the boundary effect when modeling random propagation. The overly-pessimistic behavior in the high $P_{S}$ regime is due to the asymptotic assumption made in the analytical model, which understates the spatial separation between routes when node density is not high enough.

We plot the packet interception probability as a function of the size of the black hole in Figure 16. It is clear that the interception probability increases with $R_{e}$. This trend is in line with our analytical results shown in Figure 11.

In Figure 17, we study the impact of node connectivity. The number of nodes is changed from 1000 to 3000, corresponding to changing the average node connectivity degree from 8 to 24 . It can be observed that, in general, the packet interception probabilities of the four proposed schemes do not change significantly with node connectivity. From Figure 11, we can find that even for the asymptotic case, for which the average node degree is infinite, the theoretical interception probability of the PRP scheme is about $1 \times 10^{2}$, which is slightly smaller than the simulation results. Such insensitivity to the node connectivity/density is because the packet interception probability is mainly decided by how dispersive the shares can be geographically after random propagation, i.e., how large the co-centered circles in Figure 3 can be and how the shares are distributed over these circles. As long as the nodes are uniformly distributed, the change of node density does not impact the size of these circles, nor the distribution of the shares over these circles. In contrast, the packet interception probability of H-SPREAD decreases significantly with the increase in node density, because more node-disjoint routes can be found.

In Figure 18, we slide the $\mathrm{x}$-coordinate of the source node along the line $y=0$ to evaluate the packet interception probabilities at different source locations in the network. A segmented trend can be observed: When the 
source is far away from the black hole $(-100 \leq x \leq 0)$, the closer the source is to the black hole, the smaller the packet interception probability will be. This is in line with our analytical result in Figure 12. Note that when $x=-100$ (this is at the boundary), the gap between the proposed schemes are small, because all shares can only be propagated to the right, making the random propagation process of PRP, DRP, and NRRP similar to that of MTRP. However, when the source is close to the black hole, i.e., $x \geq 0$, the trend in interception probability is reversed. This is because more and more shares are intercepted during the propagation phase. When $x=50$, which corresponds to the scenario where the source is placed right at the center of the black hole, the interception probabilities reach their maximum value. After that, they decrease quickly as the source gets farther away from the black hole. In all segments, the packet interception probabilities of the DRP, NRRP, and MTRP schemes are smaller than that of H-SPREAD.

We evaluate the average number of hops of the endto-end route as a function of the TTL value in Figure 19. This hop-count metric can be considered as an indirect measurement of the energy efficiency of the routes generated by the routing schemes. It can be observed that the hop-count under PRP, DRP, and NRRP increases linearly with $N$, while the hop-count under MTRP only increase slowly with $N$. The TTL value $N$ does not play a role in the H-SPREAD scheme. Under large $N$, e.g., when $N=25$, the randomized algorithm achieves better security performance than H-SPREAD. However, the hop-count of H-SPREAD is about $1 / 3$ of that of PRP, DRP, and NRRP, and about $1 / 2$ of that of MTRP. The relatively large hop-count in the randomized algorithms is the cost for stronger capability of bypassing black holes.

\subsubsection{Effect of the Optimization of $N$ and $M$}

In Section 3.6, we have formulated an optimization problem for PRP, which finds the most energy-efficient parameter setting $\left(N^{o}, M^{o}\right)$ among all feasible combinations of $N$ and $M$ that satisfy a given security requirement. Due to the asymptotic nature of the analytical model, the solution provided by our optimization is only optimal for PRP in an idealized setting. In this section, we use the outcome of our optimization to drive the simulation under PRP, DRP, NRRP, and MTRP, and then measure the resulting packet interception probability and the end-to-end energy consumption. The results will help us better understand the practical effect of our optimization. Our simulations are conducted as follows.

In the simulations, we assume the same target interception probability $\left(P_{S}^{(r e q)}=10^{-3}\right)$ and segmentation overhead ( $\alpha=0.1$ and $\alpha=0.01$, respectively) as in the numerical example of Section 3.7.2. Under this setting, in Section 3.7.2 we obtained all feasible combinations of $N$ and $M$. For each of these feasible $(N, M)^{\prime}$ 's, we run our simulation under PRP, DRP, NRRP, and MTRP schemes, respectively. Similar to the treatment in Section 3.7.2, the energy consumption measured in our simulations is also normalized by $L_{p} q$. The results are plotted in Figure 20 as a function of $N$ (the corresponding $M$ is omitted in the $x$-axis of the figure for brevity). The actual packet interception probability observed in our simulation is plotted in Figure 21. Two observations can be made. First, the actual energy consumption of PRP measured in the simulations presents a similar trend in $(N, M)$ to that calculated according to our analysis (see Figure 13 for comparison). As a result, the outcome of our optimization, i.e., $\left(N^{o}=10, M^{o}=28\right)$ when $\alpha=0.01$ and $\left(N^{o}=17, M^{o}=16\right)$ when $\alpha=0.1$, also achieves close-to-minimum energy consumption in the simulation. The discrepancy in the absolute value of the analytical results and the simulation outcome is not surprising, because when node density is not high enough, it takes more hops for a share to reach the destination than what is calculated under the asymptotic assumption. Second, Figure 21 shows that in the case of PRP, although the use of $\left(N^{o}, M^{o}\right)$ still achieves good energy performance, the resulting packet interception probability violates the original requirement $\left(10^{-3}\right)$. However, under DRP, NRRP, and MTRP schemes, the $\left(N^{o}, M^{o}\right)$ achieves good energy performance and in most of the time satisfies or is close to satisfying the required interception probability. This phenomenon is attributed to two reasons. First, Figures 19 and 20 have shown that DRP and NRRP present similar energy performance to PRP. This explains why using $\left(N^{o}, M^{o}\right)$ also results in good energy performance for the more advanced designs. Second, due to the overly-optimistic nature of the analysis, it is not surprising that under PRP, the actual packet interception probability under $\left(N^{o}, M^{o}\right)$ is higher than the constraint imposed on the optimization. However, because DRP, NRRP, and MTRP have better security performance than PRP, the actual packet interception probability under these algorithms is more likely to satisfy the constraint.

\subsubsection{Multi-source Case}

In Figure 22, we study the average packet interception probability of the proposed algorithms when there are multiple source nodes that are sending packets simultaneously in the system. The locations of the five source nodes used in our simulations are fixed at $(-50,0),(-50$, $25),(-50,-25),(-50,50)$, and $(-50,-50)$, respectively. These nodes are added to the simulation sequentially, yielding the average packet interception probability as a function of number of source nodes. For a given number of source nodes, the average packet interception probability is defined as the total number of packets intercepted by the eavesdropper divided by the total number of packets sent by various sources. This is in contrast to the worstcase interception probability that could happen to any single source node.

From Figure 22, it is clear that in terms of the average packet interception probability, the relative performance of various algorithms (i.e., the ranking) is similar to that 


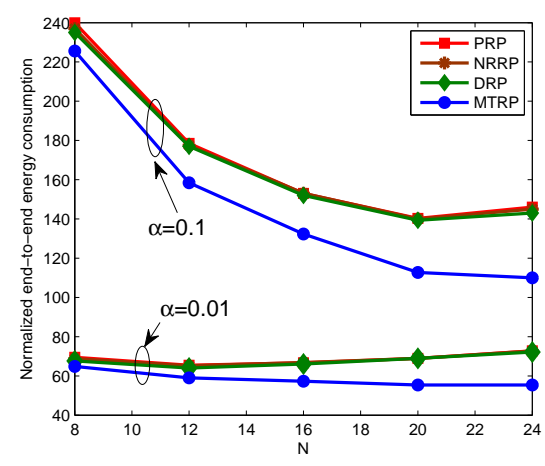

Fig. 20. Energy consumption under different $(N, M)$.

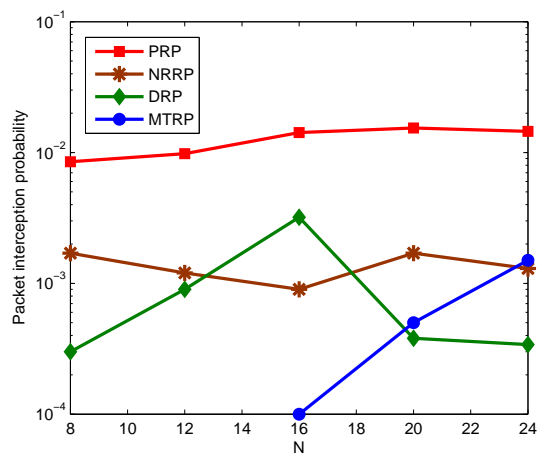

Fig. 21. Packet interception probability under different $(N, M)$.

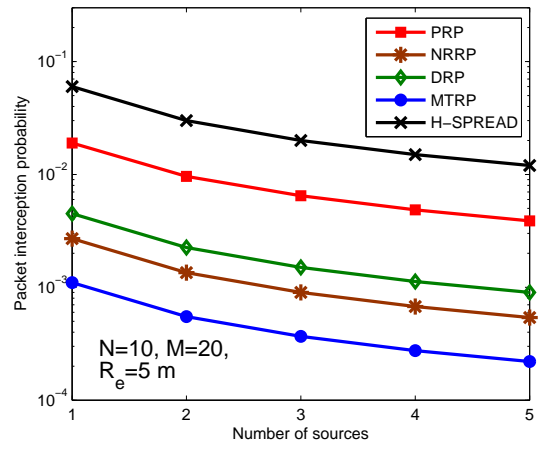

Fig. 22. Average packet interception probability vs. number of sources. under the worst-case packet interception probability. In particular, all proposed randomized multi-path routing algorithms perform better than their deterministic counterparts. This phenomenon can be explained by noting that an eavesdropper has to collect at least $T$ shares of the same information packet in order to intercept that packet. In the case of multiple source nodes, it is true that the total number of shares intercepted by the compromised node is greater than that in the singlesource case. However, these intercepted shares come from various sources. For a particular source node, the number of shares that originate from that source and are collected by the eavesdropper may not reach threshold $T$. Therefore, the larger number of intercepted shares does not necessarily indicate a higher average packet interception probability. This is especially true when the source node does not lie on the line connecting the eavesdropper and the sink, whereby most of the shares that belong to the same packet circumvent the black hole. The collinear (or near-collinear) situation contributes the most to the average packet interception probability. This is why the performance ranking in terms of the average packet interception probability under multiple sources is similar to that under the worst-case scenario under a single source.

\section{Related Work}

The concept of multi-path routing dates back to 1970s, when it was initially proposed to spread the traffic for the purpose of load balancing and throughput enhancement [15]. Later on, one of its sub-classes, path-disjoint multi-path routing, has attracted a lot of attention in wireless networks due to its robustness in combating security issues. The related work can be classified into three categories. The first category studies the classical problem of finding node-disjoint or edge-disjoint paths. Some examples include the Split Multiple Routing protocol (SMR) [8], multi-path DSR [5], and the AOMDV [13] and AODMV [24] algorithms that modify the AODV for multi-path functionality. As pointed out in [24], actually very limited number of node-disjoint paths can be found when node density is moderate and the source is far away from the destination. Furthermore, the security issue is not accounted for explicitly in this category of work.

The second category includes recent work that explicitly takes security metrics into account in constructing routes. Specifically, the SPREAD algorithm in [11], [12] attempts to find multiple most-secure and node-disjoint paths. The security of a path is defined as the likelihood of node compromise along that path, and is labeled as the weight in path selection. A modified Dijkstra algorithm is used to iteratively find the top-K most secure node-disjoint paths. The H-SPREAD algorithm [10] improves upon SPREAD by simultaneously accounting for both security and reliability requirements. The work in [6], [7] presents distributed Bound-Control and Lex-Control algorithms, which compute the multiple paths in such a way that the maximum performance degradation (e.g., throughput loss) is minimized when a single-link attack or a multi-link attack happens, respectively. The work in [23] considers the report fabrication attacks launched by compromised nodes. The work in [19] further considers selective forwarding attacks, whereby a compromised node selectively drops packets to jeopardize data availability. Both works are based on a similar cryptographic method: the secret keys used by sensor nodes are specific to their geographic locations, which limits the impact of a compromised node. Instead of relying on a cryptographic method for resolving the issue, our work mainly exploits the routing functionality of the network to reduce the chance that a packet can be acquired by the adversary in the first place. Other secure multi-path routing algorithms include SRP [16], SecMR [14], Burmester's approach [3], and AODV-MAP [21]. Among them, SRP uses end-toend symmetric cryptography to protect the integrity of the route discovery; SecMR protects against the denialof-service attack from a bounded number of collaborating malicious nodes; Burmester's method is based on the digital signatures of the intermediate nodes; AODVMAP is another modification of AODV, which can provide local bypass of the attacked nodes.

Given a set of paths that have been constructed, the 
third type of work studies the optimal way of using these paths to maximize security. For example, the Secure Message Transmission (SMT) mechanism proposed in [17] continuously updates the rating of the routes: For each successful (failed) share, the rating of the corresponding route is increased (decreased). The delivery of subsequent shares will be in favor of those routes with high ratings. The work in [4] studies two different ways of spreading an information packet into shares: secret sharing multi-path aggregation (SMA) and dispersed (message-splitting) multi-path aggregation (DMA). It shows SMA achieves better security at the cost of higher overhead, while the performance of DMA is exactly the complementary of SMA. In all above work, the multipath routing algorithms are deterministic in the sense that the same set of routes is always computed under the same topology. This weakness opens the door for a pin-pointed node-compromise or jamming attack, once the routing algorithm is acquired by the adversary.

Existing randomized multi-path routing algorithms in WSNs have not been designed with security considerations in mind, largely due to their low energy efficiency. To the best of our knowledge, the work presented in this paper fills a void in the area of secure randomized multi-path routing. Specifically, flooding is the most common randomized multi-path routing mechanism. In flooding, every node in the network receives the packet and retransmits it once. To reduce unnecessary retransmissions and improve energy efficiency, the Gossiping algorithm [9] was proposed as a form of controlled flooding, whereby a node retransmits packets according to a pre-assigned probability. It is well known that the Gossiping algorithm has a percolation behavior, in that for a given retransmission probability, either very few nodes receive the packet, or almost all nodes receive it. Parametric Gossiping was proposed in [2] to overcome the percolation behavior by relating a node's retransmission probability to its hop count from either the destination or the source. A special form of Gossiping is the Wanderer algorithm [2], whereby a node retransmits the packet to one randomly picked neighbor. When used to counter compromised-node attacks, flooding, Gossiping, and parametric Gossiping algorithms actually help the adversary intercept the packet, because multiple copies of the same secret share are dispersed to many nodes. The Wanderer algorithm has poor energy performance, because it results in long paths. In contrast, the NRRP, DRP, and MTRP schemes proposed in this paper are specifically tailored to security considerations in energyconstrained WSNs. They provide highly dispersive random routes at low energy cost without generating extra copies of secret shares.

\section{Conclusions}

Our analysis and simulation results have shown the effectiveness of the randomized dispersive routing in combating $\mathrm{CN}$ and DOS attacks. By appropriately setting the secret sharing and propagation parameters, the packet interception probability can be easily reduced by the proposed algorithms to as low as $10^{-3}$, which is at least one order of magnitude smaller than approaches that use deterministic node-disjoint multi-path routing. At the same time, we have also verified that this improved security performance comes at a reasonable cost of energy. Specifically, the energy consumption of the proposed randomized multi-path routing algorithms is only one to two times higher than that of their deterministic counterparts. The proposed algorithms can be applied to selective packets in WSNs to provide additional security levels against adversaries attempting to acquire these packets. By adjusting the random propagation and secret-sharing parameters ( $N$ and $M$ ), different security levels can be provided by our algorithms at different energy costs. Considering that the percentage of packets in a WSN that require a high security level is small, we believe that the selective use of the proposed algorithms does not significantly impact the energy efficiency of the entire system.

Our current work is based on the assumption that there is only a small number of black holes in the WSN. In reality, a stronger attack could be formed, whereby the adversary selectively compromises a large number of sensors that are several hops away from the sink to form clusters of black holes around the sink. Collaborating with each other, these black holes can form a cut around the sink and can block every path between the source and the sink. Under this cut-around-sink attack, no secret share from the source can escape from being intercepted by the adversary. Our current work does not address this attack. Its resolution requires us to extend our mechanisms to handle multiple collaborating black holes, which will be studied in our future work.

\section{REFERENCES}

[1] I. F. Akyildiz, W. Su, Y. Sankarasubramaniam, and E. Cayirci. A survey on sensor networks. IEEE Communications Magazine, 40(8):102-114, Aug. 2002.

[2] C. L. Barrett, S. J. Eidenbenz, L. Kroc, M. Marathe, and J. P. Smith. Parametric probabilistic sensor network routing. In Proceedings of the ACM International Conference on Wireless Sensor Networks and Applications (WSNA), pages 122-131, 2003.

[3] M. Burmester and T. V. Le. Secure multipath communication in mobile ad hoc networks. In Proceedings of the International Conference on Information Technology: Coding and Computing, pages 405-409, 2004

[4] T. Claveirole, M. D. de Amorim, M. Abdalla, and Y. Viniotis. Securing wireless sensor networks against aggregator compromises. IEEE Communications Magazine, pages 134-141, Apr. 2008.

[5] D. B. Johnson, D. A. Maltz, and J. Broch. DSR: the dynamic source routing protocol for multihop wireless ad hoc networks. In C. E. Perkins, editor, Ad Hoc Networking, pages 139-172. AddisonWesley, 2001.

[6] P. C. Lee, V. Misra, and D. Rubenstein. Distributed algorithms for secure multipath routing. In Proceedings of the IEEE INFOCOM Conference, pages 1952-1963, Mar. 2005.

[7] P. C. Lee, V. Misra, and D. Rubenstein. Distributed algorithms for secure multipath routing in attack-resistant networks. IEEE/ACM Transactions on Networking, 15(6):1490-1501, Dec. 2007.

[8] S. J. Lee and M. Gerla. Split multipath routing with maximally disjoint paths in ad hoc networks. In Proceedings of the IEEE ICC Conference, pages 3201-3205, 2001. 
[9] X. Y. Li, K. Moaveninejad, and O. Frieder. Regional gossip routing wireless ad hoc networks. ACM Journal of Mobile Networks and Applications, 10(1-2):61-77, Feb. 2005.

[10] W. Lou and Y. Kwon. H-spread: a hybrid multipath scheme for secure and reliable data collection in wireless sensor networks. IEEE Transactions on Vehicular Technology, 55(4):1320-1330, 2006.

[11] W. Lou, W. Liu, and Y. Fang. Spread: enhancing data confidentiality in mobile ad hoc networks. In Proceedings of the IEEE INFOCOM Conference, volume 4, pages 2404-2413, Mar. 2004.

[12] W. Lou, W. Liu, and Y. Zhang. Performance optimization using multipath routing in mobile ad hoc and wireless sensor networks. In Combinatorial Optimization in Communication Networks, pages 117-146. Kluwer, 2006.

[13] M. K. Marina and S. R. Das. On-demand multipath distance vector routing in ad hoc networks. In Proceedings of the IEEE International Conference for Network Protocols (ICNP), pages 14-23, Nov. 2001.

[14] R. Mavropodi, P. Kotzanikolaou, and C. Douligeris. Secmr- a secure multipath routing protocol for ad hoc networks. Elsevier Journal of Ad Hoc Networks, 5(1):87-99, Jan. 2007.

[15] N. F. Maxemchuk. Dispersity routing. In Proceedings of the IEEE ICC Conference, pages 41.10-41.13, 1975.

[16] P. Papadimitratos and Z. J. Haas. Secure routing for mobile ad hoc networks. In Proceedings of SCS Communication Networks and Distributed Systems Modeling and Simulation Conference (CNDS), 2002.

[17] P. Papadimitratos and Z. J. Haas. Secure dtata communication in mobile ad hoc networks. IEEE Journal on Selected Areas in Communications, 24(2):343-356, Feb. 2006.

[18] A. Perrig, R. Szewczyk, V. Wen, D. Culler, and D. Tygar. SPINS: Security protocols for sensor networks. In Proceedings of the ACM MobiCom Conference, 2001.

[19] K. Ren, W. Lou, and Y. Zhang. LEDS: Providing locationaware end-to-end data security in wireless sensor networks. In Proceedings of the IEEE INFOCOM Conference, 2006.

[20] D. R. Stinson. Cryptography, Theory and Practice. CRC Press, 2006.

[21] B. Vaidya, J. Y. Pyun, J. A. Park, and S. J. Han. Secure multipath routing scheme for mobile ad hoc network. In Proceedings of IEEE International Symposium on Dependable, Autonomic and Secure Computing, pages 163-171, 2007.

[22] A. D. Wood and J. A. Stankovic. Denial of service in sensor networks. IEEE Computer Magazine, 35(10):54-62, Oct. 2002.

[23] H. Yang, F. Ye, Y. Yuan, S. Lu, and W. Arbaugh. Toward resilient security in wireless sensor networks. In Proceedings of the ACM MobiHoc Conference, 2005.

[24] Z. Ye, V. Krishnamurthy, and S. K. Tripathi. A framework for reliable routing in mobile ad hoc networks. In Proceedings of the IEEE INFOCOM Conference, volume 1, pages 270-280, Mar. 2003.

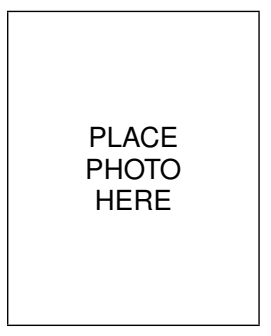

Tao Shu received the B.S. and M.S. degrees in electronic engineering from the South China University of Technology, Guangzhou, China, in 1996 and 1999, respectively, and the Ph.D. degree in electronic engineering from Tsinghua University, Beijing, China, in 2003. Currently he is a Ph.D. student at the electrical and computer engineering department at the University of Arizona, Tucson, USA. His research interests include resource allocation in wireless cellular and sensor networks, optimization of physical and MAC layers in wireless communication systems, security analysis for wireless networks, and queueing theory.

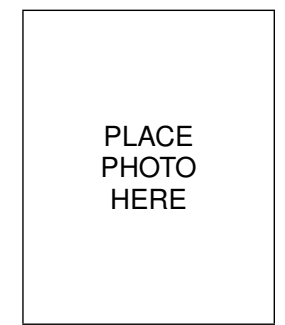

Marwan Krunz is a professor of electrical and computer engineering at the University of Arizona. He directs the wireless and networking group and is also the UA site director for Connection One, a joint NSF/state/industry IUCRC cooperative center that focuses on RF and wireless communication systems and networks. Dr. Krunz received his Ph.D. degree in electrical engineering from Michigan State University in 1995. He joined the University of Arizona in January 1997, after a brief postdoctoral stint at the University of Maryland, College Park. He previously held visiting research positions at INRIA, HP Labs, University of Paris VI, and US West (now Qwest) Advanced Technologies. His research interests lie in the fields of computer networking and wireless communications. His current research is focused on cognitive radios and SDRs; distributed radio resource management in wireless networks; channel access and protocol design; MIMO and smart-antenna systems; UWB-based personal area networks; energy management and clustering in sensor networks; media streaming; QoS routing; and fault monitoring/detection in optical networks. He has published more than 150 journal articles and refereed conference papers, and is a co-inventor on US patents. M. Krunz is a recipient of the National Science Foundation CAREER Award (1998). He currently serves on the editorial boards for the IEEE Transactions on Mobile Computing and the Computer Communications Journal. He previously served on the editorial board for the IEEE/ACM Transactions on Networking (2001-2008). He was a guest co-editor for special issues in IEEE Micro and IEEE Communications magazines. He served as a technical program chair for various international conferences, including the IEEE WoWMoM 2006, the IEEE SECON 2005, the IEEE INFOCOM 2004, and the 9th Hot Interconnects Symposium (2001). He has served and continues to serve on the executive and technical program committees of many international conferences and on the panels of several NSF directorates. He gave keynotes and tutorials, and participated in various panels at premier wireless networking conferences. He is a consultant for a number of companies in the telecommunications sector.

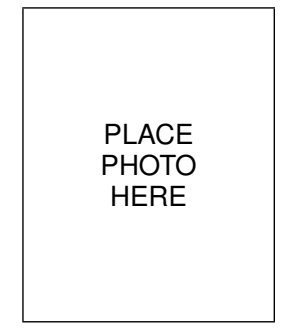

Sisi Liu received the B.S. and M.S. degree in electrical engineering from University of Electronic Science and Technology of China, Chengdu, China, in 2004 and 2007, respectively. She is currently a research assistant working toward a Ph.D degree at the Advanced networking lab, Department of Electrical \& Computer Engineering, University of Arizona, Tucson. Her research interests lie in Cognitive radio-based network, Ad Hoc networks, and wireless sensor vulnerabilities and misbehavior detection. 